\section{Metaphors for marine conservation and management: The good, the bad, and the inaccurate}

A new paper in Marine Policy discusses the importance of effective metaphors for marine conservation and policy. Metaphors are figures of speech that describe something in terms more familiar to listeners, e.g., "a blanket of snow". Good metaphors help shape understanding of something and can mobilize appropriate action. Poorly-chosen metaphors are, at best, ineffective at mobilizing support for the intended cause, and, at worst, counterproductive because they lead to oppositional behaviors or decrease the credibility of the messenger.

To be a good metaphor for marine conservation or management (or any other purpose really), a metaphor must be mapped onto something that is: 1) familiar, 2) appropriately evocative/relatable for a particular culture, and 3) scientifically accurate. Appropriate metaphors are especially important in marine conservation and management because the oceans seem remote to many people, and the scale and scope of threats to ocean ecosystems can be difficult to understand and relate to.

\section{Some good (and not so good) metaphors for marine conservation and policy}

To learn more about good and bad metaphors for marine conservation and policy, MarXiv spoke with the paper's author Alasdair Neilson. Neilson is a project manager for marine plastic pollution projects at the environmental NGO Fidra and was previously a researcher on sustainability and development projects at the University of Edinburgh. In the paper, Neilson suggests the following metaphors as effective for marine conservation and management:

- 'Fog' for background noise in the ocean

- 'Lungs of the ocean' for coral reefs

- 'Osteoporosis of the sea' for ocean acidification.

When asked about other helpful metaphors, Neilson said he'd like to see more research into metaphors of historical animal value and abundance (cod as 'gold', for example) to raise awareness of historical baselines.

According to Neilson, some potentially problematic metaphors include:

- The 'Great Pacific Garbage Patch' for marine debris in the North Pacific oceanic gyre. This metaphor is problematic because it conjures up images of macroplastics floating at the surface when the phenomenon is actually a region of increased concentration of photo-degraded plastics and other pollutants, much of which is not visible to observers. Critics have seized on this scientific inaccuracy to accuse some organizations working on marine debris issues of sensationalizing and exaggerating the severity of the phenomenon.

- 'Ocean grabbing' and 'enclosures' for the designation of marine protected areas and marine protected areas themselves. Neilson says, "More studies are analyzing marine management methods through a Marxist framework. This is important work, as the dispossession of fishing communities from their means of existence has gone unnoticed
This is a summary of: Considering the importance of metaphors for marine conservation

Accessible at: https://marxiv.org/rhefa

Author: Alasdair Neilson

Author Email: a.neilson@ed.ac.uk

Added to MarXiv: April 2018

Published: Marine Policy, 2018

Suggested Citation: Metaphors for marine conservation and management: The good, the bad, and the inaccurate. OCTO (2018). DOI: 10.17605/OSF.IO/N6YV5

See more MarXiv summaries at https://www.marxivinfo.org/ summaries

Share your research in MarXiv and the MarXiv Team may summarize it, just like this!

Step-by-step instructions on how to share your research in MarXiv are detailed at https://www.marxivinfo.org/ submission

Across all disciplines, papers available for free with a preprint (also known as "Green OA") are cited $30 \%$ more than the world average (DOI: 10.7287/peerj. preprints.3119v1) 
until relatively recently. However, when scholars use metaphorical terminology such as 'ocean grabbing' and 'enclosures' to describe the negative impacts of marine management policies (individual fishing quotas in South Africa for example), they must be sure to qualify their terminology. Such phrases, when left unqualified, can be jarring to the senses and thus hide both the complexities of the marine environment and humanity's relationship with it."

- Oceans as 'deserts'. Oceans are often compared to deserts to express their vastness and the seemingly barren nature of their surfaces. Neilson feels that this metaphor is not particularly helpful when it comes to marine conservation because "sunlit epipelagic zone surface waters are actually where the majority of organisms live. Just because we cannot see it does not mean it is not there."

In discussing good and bad metaphors, it must be stressed that good metaphors are very context-specific. A good metaphor in one region or community may be a very poor metaphor in another.

\section{How can you know if a metaphor will work?}

In the Marine Policy paper, Neilson suggests using public perception research to understand the attitudes, values, and behaviors of target audiences so that metaphors that resonate with these audiences can be constructed. When asked if this is something that marine managers or conservation practitioners could do themselves, or whether outside expertise is required, Neilson felt that there is sufficient public perception research around the marine environment - spearheaded by natural scientists using a social science toolkit - for practitioners to draw from to do this work themselves. In fact, practitioners doing this work themselves may be preferred.

Neilson says, "It is important to remember that public perception research analyzes not only how members of the public view the marine environment but also how they view marine management projects (marine protected areas. for example). Allowing a marine manager or conservation practitioner to better understand how her or his project is viewed by the local community and the wider public can only help with implementation. For this reason, I am not sure 'outsourcing' this research would be particularly helpful, as it is probably the practitioner themself who has the best understanding of the local social/ environmental context. Furthermore, on a broader note, there has long been a push in the social sciences to utilize quantitative research methods. I believe it can only be a positive step for the natural sciences to also value and employ qualitative methodologies more frequently."
The MarXiv Team

Nick Wehner,

Director of Open Initiatives, OCTO

Raye Evrard,

Project Manager, OCTO

Allie Brown,

Project Associate, ОСто

Sarah Carr,

Chief Knowledge Broker,

ОСТO

John Davis,

President, ОСTO

Correspondence

Nick Wehner

nick@octogroup.org

Financial support for MarXiv comes from the David and Lucile Packard Foundation.

The views expressed herein are those of the MarXiv Team and should not be interpreted as representing the opinions or policies of the Packard Foundation or other funders of MarXiv.

Join the MarXiv Summaries monthly newsletter at https:// oct.to/marxivsum

MarXiv is an ОСТO Initiative
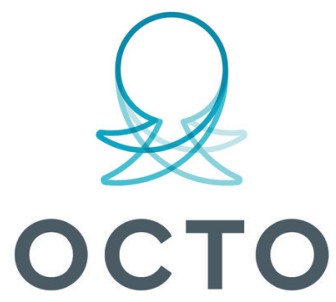

OPEN COMMUNICATIONS FOR THE OCEAN 